\title{
e-Migrinter
}

15 | 2017

Migrinter a trente ans : analyses et portraits

\section{Migrations et relations interethniques dans la ville}

Julie Garnier

\section{OpenEdition}

Journals

Édition électronique

URL : https://journals.openedition.org/e-migrinter/813

DOI : $10.4000 /$ e-migrinter.813

ISSN : 1961-9685

Éditeur

UMR 7301 - Migrinter

Référence électronique

Julie Garnier, « Migrations et relations interethniques dans la ville », e-Migrinter [En ligne], 15 | 2017,

mis en ligne le 04 mai 2017, consulté le 20 mai 2021. URL : http://journals.openedition.org/e-

migrinter/813 ; DOI : https://doi.org/10.4000/e-migrinter.813

Ce document a été généré automatiquement le 20 mai 2021.

Tous droits réservés 


\title{
Migrations et relations interethniques dans la ville
}

\author{
Julie Garnier
}

1 Présentée dès l'introduction, l'intention des chercheures ${ }^{1}$, est de combattre les idées reçues sur le racisme des classes populaires et de montrer que la complexité des situations de contact ne s'appréhende qu'en confrontant les représentations et les pratiques concrètes des groupes en présence, restituées dans leur contexte. Ces deux chercheures cherchent en effet à se défaire des stigmates qui associent les problèmes de cohabitation à la forte présence des étrangers dans la ville et à la thématique de la « distance culturelle ».

2 La sociologie des relations interethniques, qui sert de cadre théorique à cette étude, propose de porter un regard transversal sur les relations entre des populations d'origines diverses, en repartant de l'étude des lieux, des situations de contact, des circonstances, des types de liens et des motifs relationnels, à différentes échelles (du macro au microsocial). Cette approche soutient l'hypothèse que la «cohabitation pluriethnique » pensée comme une «dynamique interactive » n'est intelligible qu'en tenant compte conjointement des «rapports interethniques» et des "relations interethniques » qui se jouent dans l'espace social. L'ordre de l'ethnique se situe bien ici du côté du relationnel. Et la "cohabitation pluriethnique » apparait dans ce cadre « comme une gestion plus ou moins conflictuelle mais réglée par des rapports de force et, surtout collective, de la coexistence sociale [qui] éclaire de ce fait un des aspects de l'opposition entre domination et appropriation de l'espace, soulignée par H. Lefebvre, et participe à sa production sociale. » (p. 24).

3 Trente ans plus tard, cette étude reste d'actualité tant pour les connaissances qu'elle nous livre sur la vie sociale d'un quartier populaire immigré parisien et ses évolutions que pour l'éclairage qu'elle permet sur le renouvellement des problématiques dans le champ des migrations et des relations interethniques. Les années 1980 sont en effet marquées par le développement des travaux empiriques s'inscrivant dans cette perspective à une période où les recherches sur les migrations et les relations interethniques restent encore marginale (Streiff-Fenart, 1997; Tripier, 2004). Le 
décentrement proposé ${ }^{2}$ (Delage, 2014) dans cette recherche va ainsi ouvrir la voie à toute une série de recherches, qui porteront non plus sur le "problème de l'immigration" mais sur l'analyse des mobilités et la constitution d'espaces circulatoires transnationaux, les pratiques entreprenariales des migrants et leurs réseaux relationnels, le racisme institutionnel et la discrimination, les processus d'altérisation et d'attributions ethniques.

Cet ouvrage est également intéressant à relire pour ce qu'il nous livre sur les pratiques de recherche collaborative, mobilisant des savoirs pluridisciplinaires (notamment ceux de la géographie, de l'histoire, de la sociologie et de l'anthropologie) ainsi que différentes techniques d'enquête (relevé cartographique, analyse secondaire de statistiques, observation de longue durée, entretien approfondi, étude de parcours quotidiens, recueil de documents) dans la tradition de la sociologie urbaine inaugurée par l'École de Chicago.

5 L'enquête porte en effet sur trois secteurs urbains (Aligre centre, l'îlot Châlon, le triangle haussmannien) et aborde trois questionnements liés aux caractéristiques urbaines, aux pratiques sociales et aux échanges économiques, ce qui la distingue des monographies plus classiques de lieu. Elle met en évidence les complémentarités entre ces différents secteurs et distingue trois situations de cohabitation: dans le premier secteur (Aligre), la visibilité des immigrés dans les espaces collectifs est forte, reconnue et valorisée comme une richesse locale. Elle est liée en outre à une forme d'homogénéité sociale des habitants ainsi qu'à la densité des activités commerciales et artisanales dans le quartier, ce qui donne lieu à des formes d'entrecroisement relationnel dans un environnement pensé sur le mode du "village urbain»; dans le second secteur (le triangle haussmannien), la présence des immigrés reste invisible, elle y est en quelque sorte "absorbée par les rapports de classe", les immigrés y occupent principalement des emplois de services (p. 163), de sorte que l'espace collectif ne constitue pas un centre relationnel. «La vie sociale des immigrés ne prend pas, ici, l'espace collectif pour théâtre » (p. 108); dans le dernier secteur en cours de rénovation (îlot Châlon), la présence immigrée s'y affirme par une territorialisation communautaire, qui produit un enclavement, les excluant du reste du quartier et de la ville. Ces contrastes dans la perception de la présence immigrée et les formes d'interaction rappellent que ce qui est visible dans l'espace public ne l'est pas toujours dans l'espace résidentiel, et inversement. Ils mettent également en évidence le rôle de l'activité commerciale dans la production d'une visibilité ethnique.

6 Les chercheures s'interrogent ensuite sur le rôle et la position sociale des immigrés dans ces dynamiques en centrant l'observation sur les commerces de proximité. Les travaux nord-américains sur l'ethnic business rappellent que les migrants sont des acteurs majeurs de la (petite) production urbaine. Elles observent notamment que les rapports d'échanges marchands qui se développent dans le secteur d'Aligre " empêchent les contours communautaires de se rigidifier en frontière » (p. 144) parce qu'ils ne reposent pas sur une partition ethnique des rôles socioéconomiques mais sur une « cumulativité fonctionnelle » (p. 226), et qu'ils constituent à ce titre de " puissants fédérateurs » (p. 145). Les relations qui se développent dans ce cadre (De Rudder, 1990), loin de se limiter aux classiques rapports de subordination entre Français et immigrés comme c'est le cas dans le deuxième secteur étudié (Triangle haussmannien), s'ouvrent à des formes relationnelles plus égalitaires, qui relativisent le poids des stéréotypes et des rapports structurels dans le quartier. "Le poids des activités artisanales et 
commerciales organisent des rapports complexes de concurrence et de complémentarité, qui autorisent les affirmations collectives et en limitent les principales divisions» (p. 107). Toutefois, elles n'annulent pas les conflits. C'est en revanche, leur portée collective qui se trouve limitée, «ils ne débouchent jamais sur un clivage ethnique » (p. 146).

7 Cette précision est importante car elle rappelle combien les distorsions « entre ce qui est dit et ce qui est fait» (p. 151) sont fréquentes dans la vie sociale. Les auteures distinguent en effet trois types de discours : ceux « intégratifs » qui affirment l'unité du quartier et valorisent la présence immigrée comme une ressource dans l'esprit du « cosmopolitisme populaire» (p. 162 et p. 224); ceux qui, à l'inverse, nient cette présence pour échapper à la stigmatisation; ceux enfin, plus «stéréotypés » qui dénoncent «l'envahissement " par les étrangers (« on n'est plus chez nous») (p. 154). Ces différents discours peuvent être lus comme «autant de positions stratégiques d'habitants à l'égard de leur lieu de résidence, de son image sociale, de sa valorisation/ dévalorisation (sociale, économique, symbolique) et de son avenir. (...) Les attitudes à l'égard de la présence étrangère et de la cohabitation sont tributaires du risque de stigmatisation ethnique du quartier qui fait encourir aux habitants celui du déclassement» (p. 154 et p. 159). De sorte, que les discours sur les « Autres » n'ont, ici, " pas pour fonction de "situer" ce qu'on dit sur les relations, ils en prennent la place » (p. 151). Les auteurs concluent qu'ils existent une corrélation entre les utilités sociales, urbaines et économiques des immigrés et les types de cohabitation.

8 À l'issue de cette recherche, les chercheures montrent qu'il existe d'autres formes d'inscription spatiale des migrants dans la ville, plus ordinaires, dans lesquelles ils développent une appropriation de l'espace urbain qui produit selon les contextes, les spécificités locales et les situations d'interaction, différentes formes de visibilité, de côtoiement et de coopération; mais aussi des rapports de force et des conflits, qui apportent des éclairages sur les processus d'intégration dans la ville (Simon, 2002) et un certain savoir-faire pluraliste, qui n'est toutefois jamais acquis.

\section{BIBLIOGRAPHIE}

(2014) Hommes et Migrations, $n^{\circ} 1308$, (Les Paris des migrants).

Delage, Mathieu (2014) Paris et ses migrants : dans les pas de Michelle Guillon, Hommes et migrations, $\mathrm{n}^{\circ} 1308,1 \mathrm{p}$.

Guillon, Michelle ; Taboada-Leonetti, Isabelle (1986) Le triangle de Choisy. Un quartier chinois à Paris, Paris, L'Harmattan, 212 p. (CIEMI).

Guillon, Michelle ; Taboada-Leonetti, Isabelle (1988) Les immigrés des beaux quartiers. La communauté espagnole dans le XVIe, Paris, L'Harmattan, 210 p. (Migrations et changements).

De Rudder, Véronique ; Guillon, Michelle (1987) Autochtones et immigrés en quartier populaire. D'Aligre à l'îlot Châlon, Paris, L'Harmattan, 234 p. (CIEMI). 
De Rudder, Véronique (1990) La cohabitation pluriethnique et ses enjeux, Migrants-Formation, $\mathrm{n}^{\circ} 80$, pp. 68-90.

Streiff-Fenart, Jocelyne (1997) Les recherches interethniques en France, le renouveau ?, MigrantsFormation, $\mathrm{n}^{\circ} 109$, pp. 48-65.

Simon, Patrick (2002) L'intégration et les relations interethniques dans la ville in Roncin, C. ; Penven, A. ; Bonny, Y. (dir.) Au cœur de la cité. Vivre ensemble, travailler, s'engager, Rennes, Presses universitaires de Rennes, pp. 73-86.

Tripier, Maryse (2004) L'immigré, analyseur de la société, (note critique), Terrains \& travaux, $\mathrm{n}^{\circ} 7$, vol. 2, pp. 173-185.

\section{NOTES}

1. Véronique De Rudder était chargée de recherches au CNRS, membre de l'URMIS, responsable et directrice de publication du réseau REMISIS de 1985 à 2014. Michelle Guillon était professeure de géographie à l'Université de Poitiers, membre de Migrinter, directrice de la Revue européenne des migrations internationales (REMI) de 1993 à 2006.

2. Ces deux chercheures sont pionnières dans l'émergence d'une géographie sociale de l'immigration dans la métropole parisienne et d'une sociologie des relations interethniques et du racisme en France.

\section{RÉSUMÉS}

« Autochtones et immigrés en quartier populaire. D’Aligre à l'îlot Châlon » est le second ouvrage d'une série d'études conduites dans trois quartiers parisiens à la fin des années 1980 , avec le soutien de la mission de la recherche urbaine du ministère de l'Urbanisme et du Logement. Publié en 1987 par Véronique De Rudder, en collaboration avec Michelle Guillon, l'ouvrage aborde la question des relations interethniques dans la ville en partant du postulat que toutes les situations résidentielles urbaines sont des situations de "cohabitation pluriethnique ». Cette situation de cohabitation, «loin de former un système dichotomique, est construite par des relations « entre des groupes multiples, fluctuants, difficiles à définir avec précision, traversés de multiples clivages » (J. Mantovani et O. Saint-Raymond (1984) cité par De Rudder, 1990, p. 70).

\section{INDEX}

Index géographique : France, Paris

Mots-clés : commerce ethnique, quartier, pratique sociale, réseaux communautaires, étude de cas 
AUTEUR

JULIE GARNIER

Maître de conférences en sociologie, Laboratoire CITERES - UMR 7324, CNRS / Université François Rabelais (Tours), Équipe Construction Politique et Sociale des Territoires (CoST)

julie.garnier@univ-tours.fr 